\title{
Características psicométricas do Questionário Newcastle de Conhecimento em Asma (NAKQ) para pais de crianças com asma
}

\author{
Psychometric characteristics of the Newcastle Asthma Knowledge Questionnaire (NAKQ) \\ for parents of children with asthma
}

\author{
Cristian Roncada ${ }^{1,3} \bowtie$, Luísa Carolina Bischoff ${ }^{2}$, Bianca Martininghi Bugança ${ }^{2}$, Karina Soldera ${ }^{2}$, \\ Thiago de Araujo Cardoso ${ }^{3}$, Paulo Márcio Pitrez ${ }^{3}$ \\ ${ }^{1}$ Curso de Educação Física da Faculdade da Serra Gaúcha (FSG). Caxias do Sul, RS. \\ ${ }^{2}$ Curso de Biomedicina da Faculdade da Serra Gaúcha (FSG). Caxias do Sul, RS. \\ ${ }^{3}$ Núcleo de Educação em Saúde da Criança (NESC), Instituto de Pesquisas Biomédicas (IPB), Pontifícia Universidade Católica do Rio Grande do Sul (PUCRS). \\ Porto Alegre, RS.
}

\section{RESUMO}

OBJETIVOS: Analisar as características psicométricas identificadas pelo questionário de conhecimento em asma Newcastle Asthma Knowledge Questionnaire (NAKQ) em pais de crianças com asma.

MÉTODOS: Foram recrutados pais de crianças com asma em acompanhamento ambulatorial com pneumologistas pediátricos. Pais de crianças sem diagnóstico de asma foram recrutados em escolas públicas da mesma cidade. O questionário NAKQ possui 31 itens, sendo 25 questões com respostas "verdadeiro" ou "falso", e seis questões abertas. A pontuação mínima de conhecimento é de 0 (zero) pontos e a pontuação máxima é de 31 pontos. Além disso, o questionário recebe um ponto de corte $\geq 21$ pontos para níveis satisfatórios (adequado) e $<21$ para níveis insatisfatórios (inadequado). Para a análise de precisão do instrumento foram adotados três critérios: a) consistência interna por meio do coeficiente alfa de Cronbach $(\alpha \mathrm{C})$; b) coeficiente de correlação intraclasse (CCI); e b) teste-reteste por meio do coeficiente Kappa. Para a validação foi analisado o critério de validade corrente.

RESULTADOS: Cento e cinquenta e quatro pais participaram do estudo, sendo 62 (40,3\%) do grupo de asmáticos e 92 (59,7\%) do grupo controle, com média de idade $35,6 \pm 10,03$ anos, sendo $132(85,7 \%)$ do sexo feminino. O ensino médio completo foi a escolaridade mais prevalente $(n=72 ; 46,8 \%)$. O coeficiente $\alpha \mathrm{C}$ foi de 0,72 e 0,70 , para os grupos de pais de asmáticos e pais de crianças não asmáticas, respectivamente. O CCI foi de 0,703 (IC95\%: 0,042-0,111) para pais de asmáticos e 0,687 (IC95\%: 0,588-0,772) para pais de crianças sem asma. O grau de consistência nas respostas para cada item sobre a administração repetida do questionário, com aplicação do índice de Kappa, obteve valores aceitáveis em 25 (80,65\%) questões, sendo oito $(25,81 \%)$ classificadas como "boa" e 17 (54,84\%) como "muito boa".

CONCLUSÕES: O NAKQ apresentou bons índices de consistência interna e reprodutibilidade, demostrando ser um instrumento preciso e válido para mensurar o nível de conhecimento sobre a doença em pais de crianças brasileiras com asma.

DESCRITORES: asma brônquica; pais; cuidadores; criança; educação em saúde.

\section{ABSTRACT}

AIMS: To analyze the psychometric characteristics identified by the Newcastle Asthma Knowledge Questionnaire (NAKQ) in parents of children with asthma.

METHODS: Parents of children with asthma were recruited in outpatient follow-up with pediatric pulmonologists. Parents of children with no asthma diagnosis were recruited from public schools in the same city. The NAKQ has 31 items, being 25 questions with answers "true" or "false", and six open questions. The minimum score for knowledge is of 0 (zero) points with a maximum score of 31 points. In addition, the questionnaire is given a cutting point $\geq 21$ points to satisfactory levels (suitable) and 21 for unsatisfactory levels $<$ (inappropriate). For the analysis of precision of the instrument, we adopted three criteria: a) internal consistency through the alpha Cronbach ( $\alpha \mathrm{C})$ coefficient; b) intraclass correlation coefficient (ICC); and b) test-retest using the Kappa coefficient. For the questionnaire validation, we analyzed the current validity criteria.

RESULTS: One hundred and fifty four parents participated in this study, with $62(40.3 \%)$ children with asthma and $92(59.7 \%)$ without asthma, with a mean of $35.6 \pm 10.03$ years and $132(85.7 \%)$ females. High school was the most prevalent education level ( $\mathrm{n}=72 ; 46.8 \%)$. The $\alpha \mathrm{C}$ coefficient was 0.72 and 0.70 , for the group of parents of asthmatics and of non-asthmatic children, respectively. The ICC was 0.703 (CI95\%: 0.042-0.111) for parents of asthmatics and 0.687 (CI95\%: 0.588-0.772) for parents of non-asthmatic. The degree of consistency in responses to each item on the repeated questionnaires, with application of the Kappa index, obtained acceptable values in 25 (80.65\%), with eight issues (25.81\%) classified as "good" and 17 (54.84\%) as "very good".

CONCLUSIONS: The NAKQ presented good internal consistency and repeatability indices, showing to be a precise and valid tool for measuring the level of knowledge about the disease in parents of Brazilian children with asthma.

KEY WORDS: asthma; parents; caregivers; child; health education.

Recebido: novembro, 2016

Aceito: abril, 2017

Publicado: maio, 2017 
Abreviaturas: $\alpha \mathrm{C}$, coeficiente alfa de Cronbach; CCI, coeficiente de correlação intraclasse; GINA, Global Initiative for Asthma; ISPOR-TCA, International Society for Pharmacoeconomics and Outcomes Research-Translation and Cultural Adaptation; NAKQ, Newcastle Asthma Knowledge Questionnaire.

\section{INTRODUÇÃO}

A asma é a doença crônica mais comum na criança, com elevada prevalência mundial [1]. Recentemente, tem sido salientada a importância do conhecimento sobre a doença por parte das pessoas envolvidas, pois a falta do controle e da adesão ao tratamento resultam em elevada morbidade [2-4]. Exceto em crianças com asma leve, o tratamento medicamentoso deve ser feito de forma contínua, mas a falta de conhecimento dos pais sobre a doença, incluindo a não compreensão da sua gravidade e informações inadequadas sobre os medicamentos, compromete muitas vezes o seu manejo correto e eficaz [5]. Para isso, o acompanhamento do paciente por profissionais de saúde, com programas de educação, tem exercido papel central no controle da asma $[6,7]$.

$\mathrm{Na}$ criança com asma, os pais são personagens centrais no manejo clínico, gestão de medicamentos e tratamento. A avaliação sistemática do nível de conhecimento dos pais acerca da doença é fundamental para auxiliar o médico na tomada de decisões sobre quais assuntos são mais importantes à serem abordados durante cada consulta [8]. Dessa forma, os questionários de avaliação de conhecimento em asma representam um importante método para demonstrar se as intervenções educacionais estão sendo efetivas [9]. Considerando que existem diversos instrumentos para avaliação dos níveis de conhecimento sobre a doença, escolher o mais adequado pode ser uma tarefa difícil. Uma revisão sistemática [10] demonstrou que de 22 questionários com essa finalidade, a maioria não apresentava metodologia de validação satisfatória, conforme critérios de Terwee et al. [11]. Além disso, não foi encontrado nenhum instrumento específico para avaliação do conhecimento sobre asma direcionado aos pais de crianças com asma no idioma Português brasileiro.

O Newcastle Asthma Knowledge Questionnaire (NAKQ) é um questionário internacional desenvolvido para mensurar o nível de conhecimento sobre a doença em pais de crianças com asma. Possui versões traduzidas e validadas para a língua inglesa [12] e espanhola [13]. No Brasil, esse questionário possui validação linguística e psicométrica somente para profissionais em saúde [14], não tendo sido analisado e validado no público originalmente projetado (pais de crianças com asma). Assim, este estudo tem como objetivo analisar as características psicométricas do NAKQ em pais de crianças com asma, na língua portuguesa do Brasil.

\section{MÉTODOS}

Foi realizado um estudo transversal, entre abril de 2015 e março de 2016, no qual foram recrutados sujeitos de duas populações distintas de pais de crianças e adolescentes com idade entre seis e 17 anos. A primeira população consistia em pais de crianças com diagnóstico médico de asma, e a segunda, de pais de crianças que nunca tiveram diagnóstico de asma. Os locais de recrutamento foram as escolas da rede pública da cidade de Porto Alegre e o ambulatório de um centro de referência em pneumologia pediátrica localizado na mesma cidade. O estudo foi aprovado pelo Comitê de Ética em Pesquisa da Pontifícia Universidade Católica do Rio Grande do Sul sob o parecer de número 379.864 .

Os sujeitos recrutados foram divididos em dois grupos: grupo $\mathrm{A}$ - pais de crianças com diagnóstico de asma; grupo B - pais de crianças sem diagnóstico de asma. O diagnóstico de asma, bem como a classificação do controle da asma (controlada, parcialmente controlada e não controlada), foram baseados nos critérios da diretriz internacional da Global Initiative for Asthma (GINA) [15, 16].

Os sujeitos do grupo A (asma) foram selecionados na sala de espera do ambulatório do centro de referência, no dia agendado para a consulta médica regular com o pneumologista pediátrico, através de abordagem direta do entrevistador e explicação do estudo. Se a pessoa (mãe ou pai da criança/adolescente) concordasse em participar, era convidada a assinar o Termos de Consentimento Livre e Esclarecido. Após isso, eram aplicados os testes e os questionários. As crianças/adolescentes também assinaram o Termo de Assentimento e responderam aos testes pertinentes.

Para o grupo B (controle), os sujeitos foram selecionados nas escolas públicas da região com os mesmos procedimentos, após a informação de que a criança não tinha diagnóstico médico de asma. Os pais do grupo controle não poderiam ter nenhum filho com asma.

Os critérios de exclusão consistiram em crianças e adolescentes com limitações cognitivas, motoras ou com outras doenças crônicas que pudessem comprometer a avaliação do controle da doença. 
O instrumento NAKQ, desenvolvido por Fitzclarence e Henry [12], na Austrália, foi escolhido para este estudo por ser o mais aplicado em estudos sobre o assunto, tendo sido validado em 1990. O NAKQ possui tradução linguística e cultural para o Espanhol desde 2009, realizada por Praena et al. [13], e recentemente para o Português do Brasil somente para profissionais da área da saúde (médicos e não médicos), realizada pelo nosso grupo [14]. O questionário possui 31 itens, com 25 questões com respostas "verdadeiro" ou "falso", e seis questões abertas. As respostas corretas recebem valor $1 \mathrm{e}$ as incorretas recebem valor 0 . Portanto, a pontuação mínima de conhecimento é de 0 (zero) pontos e a pontuação máxima é de 31 pontos. Além disso, o questionário recebe um ponto de corte $\geq 21$ pontos para níveis satisfatórios (adequado) e $<21$ para níveis insatisfatórios (inadequado).

Para a validação do NAKQ foi seguida a metodologia sugerida pelo ISPOR-TCA (International Society for Pharmacoeconomics and Outcomes Research-Translation and Cultural Adaptation) Task Force [17], a mesma adotada por Praena et al. [13]. Em virtude da validação de tradução e retro-tradução (Inglês/Português) já ter sido realizada por Cidade et al. [14], adotamos apenas a validade concorrente para análise dos dados coletados [18].

Para análise de precisão (fidedignidade) da consistência interna foi aplicado o coeficiente alfa de Cronbach $(\alpha C)$ para cada dimensão e para o escore total das dimensões, onde valores $>0,60$ e $<0,95$ são considerados válidos e aceitáveis $[19,20]$, além do coeficiente de correlação intraclasse (CCI) [10], sendo adotado um valor de $\mathrm{p}<0,05$ como estatisticamente significativo.

Para fins de reprodutibilidade foram adotados os mesmos critérios utilizados por Praena et al. [13] e aplicados teste e reteste em 20 participantes (10 para cada grupo), com intervalo entre três e quatro semanas, sem nenhuma intervenção de educação em asma. Para análise de concordância foi aplicada a análise de Kappa, utilizando os critérios pelos quais valores $\leq 0,2$ são considerados pobres, entre $0,21-0,40$ fracos, entre 0,41-0,60 moderados, entre 0,61-0,80 bons, e $\geq 0,81$ muitos bons. Desta forma, consideramos valores $>0,60$ como aceitáveis e $>0,80$ como ótima concor dância [11].

Os demais resultados dos questionários foram submetidos aos seguintes testes: qui-quadrado para comparação da pontuação de acertos entre os grupos (asma e controles) e teste $t$ independente para comparação entre as médias da pontuação total. Os dados categóricos foram apresentados por frequências absolutas e relativas e os valores contínuos por média e desvio-padrão.

Os dados foram armazenados em uma planilha do Microsoft Access versão 2013 e analisados pelo software estatístico IBM SPSS versão 20.

\section{RESULTADOS}

Foram incluídos no estudo um total de 154 sujeitos, sendo 62 pais de crianças asmáticas e 92 de crianças não asmáticas, idade média de 35,6 610,03 anos, 132 $(85,7 \%)$ do sexo feminino e $72(46,8 \%)$ pais com ensino médio completo. Esses dados sociodemográficos não apresentaram diferenças significativas entre os grupos, indicando ser uma amostra homogênea. No geral, somente 47 pais $(30,5 \%)$ demonstraram nível aceitável de conhecimento sobre asma através da aplicação do NAKQ. Além disso, a frequência de pais com escore acima de 21 pontos foi maior no grupo de pais de asmáticos (41,9\% vs. 22,8\%; p=0,010). As características dos sujeitos nos grupos estudados e a pontuação geral do NAKQ são apresentadas na Tabela 1.

Dos 31 pontos do questionário, a pontuação média do nível de conhecimento dos 154 participantes foi de $18,1 \pm 4,1$ pontos. Entre os grupos estudados, os pais de crianças com asma obtiveram uma média maior de pontos $(19,3 \pm 3,9)$, quando comparados ao grupo controle $(17,2 \pm 4,0)(\mathrm{p}=0,001)$.

Foi aplicado o $\alpha \mathrm{C}$ tanto para a pontuação do escore total (31 itens), quanto para pontuação do escore por itens. $\mathrm{Na}$ análise de pontuação do escore total, o instrumento obteve valor de $\alpha \mathrm{C}$ igual a 0,71 pontos. Para a análise por itens, os valores de $\alpha \mathrm{C}$ foram de 0,62 para pontuação mínima e de 0,68 para pontuação máxima. Quando o instrumento foi analisado por grupos, a pontuação do escore total para o grupo asma foi de 0,72 , e para o grupo controle de 0,70 (Tabela 2).

Para o CCI o valor total entre os dois grupos foi de 0,705 (IC95\%: 0,634-0,768); verificando cada grupo individualmente os resultados foram: 0,703 (IC95\%: 0,042-0,111) para pais de asmáticos e 0,687 (IC95\%: $0,588-0,772)$ para o grupo controle $(\mathrm{p}<0,001)$.

O grau de consistência nas respostas para cada item sobre a administração repetida do questionário, com aplicação do índice de Kappa, obteve valores aceitáveis em 25 das 31 questões $(80,65 \%)$, sendo oito classificadas como "boa" $(25,81 \%)$ e 17 como "muito boa" $(54,84 \%)$ (Tabela 3$)$. 
Tabela 1. Características da amostra de pais e crianças com e sem diagnóstico de asma e pontuação obtida no Newcastle Asthma Knowledge Questionnaire (NAKQ). Porto Alegre, Rio Grande do Sul, 2016.

\begin{tabular}{|c|c|c|c|c|c|}
\hline & \multicolumn{2}{|c|}{ Pais de crianças com asma $(n=62)$} & \multicolumn{2}{|c|}{ Pais de crianças sem asma $(n=92)$} & \multirow{2}{*}{$\mathbf{p}$} \\
\hline & n (\%) & Média \pm DP & n (\%) & Média \pm DP & \\
\hline \multicolumn{6}{|l|}{ Dados das crianças } \\
\hline Idade, anos & & $10,2 \pm 3,1$ & & $10,4 \pm 4,0$ & 0,722 \\
\hline Sexo feminino & $33(53,2)$ & & $48(52,1)$ & & \\
\hline \multicolumn{6}{|c|}{ Controle da asma (GINA-2016) } \\
\hline Controlada & $21(33,9)$ & & & & \\
\hline Parcialmente controlada & $23(37,1)$ & & & & \\
\hline Não controlada & $18(29,0)$ & & & & \\
\hline \multicolumn{6}{|l|}{ Dados dos pais } \\
\hline Idade, anos & & $36,2 \pm 9,2$ & & $35,2 \pm 10,6$ & 0,547 \\
\hline Sexo feminino & $57(91,9)$ & & $75(81,5)$ & & 0,071 \\
\hline \multicolumn{6}{|l|}{ Escolaridade } \\
\hline Ensino básico & $9(14,5)$ & & $8(8,7)$ & & \multirow{4}{*}{0,063} \\
\hline Ensino fundamental & $12(19,4)$ & & $21(22,8)$ & & \\
\hline Ensino médio & $36(58,1)$ & & $52(56,5)$ & & \\
\hline Ensino superior & $5(8,1)$ & & $11(12,0)$ & & \\
\hline Quantidade de filhos & & $1,5 \pm 3,9$ & & $1,0 \pm 0,9$ & $0,002^{*}$ \\
\hline Pontuação no NAKQ & & $19,3 \pm 3,9$ & & $17,2 \pm 4,0$ & $<0,001^{*}$ \\
\hline
\end{tabular}

DP, desvio padrão; GINA, Global Initiative for Asthma; NAKQ, Newcastle Asthma Knowledge Questionnaire.

Tabela 2. Avaliação e comparação entre o percentual de acertos e avaliação pelo coeficiente alfa de Cronbach ( $\alpha$ C) por itens, entre pais de crianças com asma e pais de crianças sem asma (controles).

\begin{tabular}{|c|c|c|c|c|c|c|c|c|}
\hline \multicolumn{2}{|l|}{ Perguntas do questionário } & \multicolumn{3}{|c|}{$\begin{array}{l}\text { Pais de crianças com asma } \\
n=62\end{array}$} & \multicolumn{3}{|c|}{$\begin{array}{l}\text { Controles } \\
\mathrm{n}=92\end{array}$} & \multirow[t]{2}{*}{$\mathbf{p}$} \\
\hline & & n & $\%$ & $\alpha \mathrm{C}$ & n & $\%$ & $\alpha \mathrm{C}$ & \\
\hline 1. Quais são os três sintomas principais da asma? & R1 & 27 & 43,5 & 0,65 & 30 & 32,6 & 0,65 & 0,11 \\
\hline $\begin{array}{l}\text { 2. Uma em cada } 10 \text { crianças terá asma em algum momento de sua } \\
\text { infância. }\end{array}$ & $\mathrm{V}$ & 50 & 80,6 & 0,66 & 65 & 70,7 & 0,67 & 0,11 \\
\hline 3. As crianças com asma possuem vias aéreas muito mais sensíveis. & $\mathrm{V}$ & 54 & 87,1 & 0,65 & 78 & 84,8 & 0,65 & 0,44 \\
\hline $\begin{array}{l}\text { 4. Se uma criança em uma família tem asma então é quase certo que } \\
\text { os seus irmãos e irmãs também tem. }\end{array}$ & $\mathrm{F}$ & 35 & 56,5 & 0,65 & 75 & 81,5 & 0,64 & $<0,01$ \\
\hline $\begin{array}{l}\text { 5. A maioria das crianças com asma fica mais encatarrada quando bebe } \\
\text { leite de vaca. }\end{array}$ & $\mathrm{F}$ & 52 & 83,9 & 0,65 & 37 & 40,2 & 0,63 & $<0,01$ \\
\hline $\begin{array}{l}\text { 6. Anote todas as coisas que você sabe que provocam uma crise de asma } \\
\text { (às vezes chamadas de desencadeantes) }\end{array}$ & R6 & 55 & 88,7 & 0,64 & 70 & 76,1 & 0,65 & 0,04 \\
\hline $\begin{array}{l}\text { 7. Durante uma crise de asma o chiado no peito (ou apito ou miado } \\
\text { de gato) pode acontecer devido à contração dos músculos da parede } \\
\text { dos brônquios. }\end{array}$ & V & 42 & 67,7 & 0,67 & 64 & 69,6 & 0,63 & 0,47 \\
\hline $\begin{array}{l}\text { 8. Durante uma crise de asma o chiado pode acontecer devido à } \\
\text { inflamação da parte interna dos brônquios. }\end{array}$ & V & 50 & 80,6 & 0,64 & 63 & 68,5 & 0,64 & 0,07 \\
\hline 9. A asma causa danos ao coração & $\mathrm{F}$ & 24 & 38,7 & 0,68 & 25 & 27,2 & 0,67 & 0,09 \\
\hline $\begin{array}{l}\text { 10. Escreva o nome de dois tratamentos (medicações) para a asma que } \\
\text { se devem tomar regularmente todos os dias para prevenir crises } \\
\text { de asma. }\end{array}$ & R10 & 44 & 71,0 & 0,65 & 38 & 41,3 & 0,64 & $<0,01$ \\
\hline $\begin{array}{l}\text { 11. Escreva o nome de três tratamentos (medicamentos) que são úteis } \\
\text { durante uma crise de asma. }\end{array}$ & R11 & 50 & 80,6 & 0,63 & 42 & 45,7 & 0,64 & $<0,01$ \\
\hline $\begin{array}{l}\text { 12. Os antibióticos são uma parte importante do tratamento para a } \\
\text { maioria das crianças com asma. }\end{array}$ & $\mathrm{F}$ & 27 & 43,5 & 0,62 & 26 & 28,3 & 0,65 & 0,04 \\
\hline $\begin{array}{l}\text { 13. A maioria das crianças com asma não deveria consumir produtos } \\
\text { derivados do leite. }\end{array}$ & $\mathrm{F}$ & 43 & 69,4 & 0,63 & 42 & 45,7 & 0,64 & $<0,01$ \\
\hline 14. As vacinas para asma curam a doença. & $\mathrm{F}$ & 47 & 75,8 & 0,64 & 82 & 89,1 & 0,64 & 0,02 \\
\hline
\end{tabular}


Tabela 2 (conclusão)

Pais de crianças com asma Controles

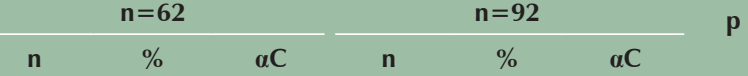

15. Se uma pessoa morre de uma crise de asma isso geralmente significa que a crise deve ter começado tão rápido que não houve tempo para iniciar qualquer tratamento.

16. As pessoas com asma geralmente têm "problema dos nervos".

17. A asma é infecciosa, ou seja, você pode se contagiar através de outra pessoa.

n

F 31

50,0

0,64

41

$44,6 \quad 0,65$

0,30

F $\quad 35$

$56,5 \quad 0,64 \quad 43$

46,7

0,65

0,15

$\begin{array}{llllllll}\text { F } & 60 & 96,8 & 0,64 & 89 & 96,7 & 0,64 & 0,68\end{array}$

18. Os medicamentos inalados para asma, por exemplo salbutamol ou fenoterol, têm menos efeitos colaterais do que comprimidos/xaropes.

19. Corticoides orais (prednisona ou prednisolona) usados por curta duração geralmente provocam importantes efeitos colaterais.

20. Alguns tratamentos para a asma (como salbutamol ou fenoterol) fazem mal para o coração.

21. Uma criança de 5 anos sofre uma crise de asma e usa 2 jatos de salbutamol inalado (bombinha). Após 5 minutos ele não está melhor. Escreva algumas razões possíveis pelas quais isso aconteceu.

V

V

F $\quad 30$

24,2

0,65

23

25,0

0,63

F $\quad 24$

48,4

0,62

35

$38,0 \quad 0,64$

0,13

. Durante uma crise de asma que você está tratando em casa a sua criança precisa nebulizar ou usar bombinha de salbutamol a cada 2 horas. Ficou melhor mas depois de 2 horas ainda está com falta de ar. Considerando-se que a criança não piorou é correto continuar o tratamento a cada 2 horas.

23. Escreva formas de ajudar a prevenir crises de asma durante o exercício.

R21

24

0,63

45

48,9

$0,63 \quad 0,14$

R21

14

22,6

0,66

20

21,7

0,64

0,52

4. As crianças com asma se tornam viciadas em seus medicamentos para asma.

25. A natação é o único exercício apropriado para asmáticos.

26. O fato dos pais serem fumantes pode piorar a asma da criança.

27. Com o tratamento adequado a maioria das crianças com asma deve ter uma vida normal sem restrição de suas atividades.

28. A melhor maneira de medir a gravidade da asma da criança é o médico ouvir o peito dela.

29. A asma é geralmente mais problemática à noite do que durante o dia.

30. A maioria das crianças com asma tem problemas de baixo crescimento.

31. Crianças com sintomas de asma frequente devem usar medicamentos preventivos.

Escore total

\begin{tabular}{llllllll} 
F & 14 & 22,6 & 0,68 & 13 & 14,1 & 0,64 & 0,13 \\
R23 & 13 & 21,0 & 0,65 & 22 & 23,9 & 0,64 & 0,41 \\
F & 33 & 53,2 & 0,64 & 57 & 62,0 & 0,63 & 0,18 \\
F & 36 & 58,1 & 0,63 & 63 & 68,5 & 0,62 & 0,13 \\
V & 57 & 91,9 & 0,64 & 83 & 90,2 & 0,64 & 0,48 \\
V & 55 & 88,7 & 0,64 & 81 & 88,0 & 0,65 & 0,56 \\
F & 22 & 35,5 & 0,64 & 35 & 38,0 & 0,65 & 0,44 \\
V & 48 & 77,4 & 0,65 & 68 & 73,9 & 0,65 & 0,38 \\
F & 52 & 83,9 & 0,65 & 55 & 59,8 & 0,64 & $<0,01$ \\
\hline V & 59 & 95,2 & 0,65 & 73 & 79,3 & 0,65 & $<0,01$ \\
$\geq 21$ & 26 & 41,9 & 0,72 & 21 & 22,8 & 0,70 & 0,01 \\
\hline
\end{tabular}

Controle, grupo de pais de crianças sem asma. V, verdadeira; F, falsa; R1, tosse, chiado ou sibilo e falta de ar ou dispneia; R6, alergia, resfriado e exercício; R10, dois medicamentos de: corticoide inalado, antileucotrieno ou beta-2 adrenérgico de longa ação; R11, dois medicamentos de: beta-2-adrenérgicos de curta ação, brometo de ipratrópio ou corticoide oral; R21, dois itens de: dose insuficiente, crise muito grave, técnica inalatória ruim ou dispositivo vazio; R23, dois itens de: beta-2 de ação curta antes do exercício, melhorar o controle da asma (profilaxia medicamentosa adequada) ou aquecimento físico. Escore total: <21 pontos, níveis inadequados; $\geq 21$ pontos, níveis aceitáveis.

Tabela 3. Avaliação do índice de Kappa (teste e reteste) aplicado em 20 participantes: pais de asmáticos $(n=10)$ e controles $(n=10)$, com intervalo de três a quatro semanas, sem aplicação de intervenção em asma.

\begin{tabular}{lccc}
\multicolumn{1}{c}{ Grau } & Kappa & n $(\%)$ & No da pergunta* \\
\hline Pobre & $\leq 0,2$ & $2(6,45)$ & 21,23 \\
Fraco & $0,21-0,40$ & $1(3,23)$ & 10 \\
Moderado & $0,41-0,60$ & $3(9,68)$ & $1,6,13$ \\
Bom & $0,61-0,80$ & $8(25,81)$ & $3,5,7,9,15$, \\
& & & $18,28,29$ \\
Muito bom & & & $2,4,8,11,12$, \\
& & $17(54,84)$ & $14,16,17,19$, \\
& & & $20,22,24,25$, \\
\end{tabular}

* Correspondem aos itens/perguntas do questionário da Tabela 2.

\section{DISCUSSÃO}

Avaliar os níveis de conhecimento em asma de pacientes, pais e profissionais em saúde tem se tornado uma ferramenta essencial para melhorar o manejo da doença [21]. Entender as limitações e necessidades dos pais auxilia o médico a planejar os tópicos a serem abordados durante as consultas, viabilizando mais momentos dedicados à educação em saúde, diminuindo as dúvidas e desmistificando conceitos errados [22]. Além disso, mensurar o nível de conhecimento em asma proporciona uma visão epidemiológica ampla acerca dos rumos da doença nos diversos níveis das 
políticas de saúde, demonstrando aos gestores quais são as regiões que necessitam mais investimentos e estrutura educacional [9].

Para a avaliação dos níveis de conhecimento em asma são utilizados instrumentos específicos e, atualmente, existem 22 questionários padronizados e validados para tal finalidade, porém, apenas um deles foi desenvolvido por pesquisadores brasileiros [23] e mesmo assim, direcionado para adultos com asma. Outro questionário amplamente aplicado é o NAKQ, que foi traduzido e validado recentemente pelo nosso grupo de pesquisa, sendo aplicado a profissionais da área da saúde [14]. Assim, o presente estudo avaliou o NAKQ quanto a suas propriedades psicométricas em pais de crianças brasileiras com asma.

O NAKQ tem sido utilizado também para outros públicos, como profissionais da saúde ou professores de crianças com asma em fase escolar [13, 24]. A versão brasileira do NAKQ para pais de crianças com asma demonstrou consistência interna adequada por meio da análise do alfa de Cronbach $(\alpha \mathrm{C}=0,71)$, com valores muito próximos aos encontrados na versão original $(\alpha \mathrm{C}=0,69)$, além das versões em Espanhol $(\alpha C=0,72)$ e da versão em Português aplicada a profissionais em saúde $(\alpha C=0,69)$ [25]. A interpretação estatística do $\alpha \mathrm{C}$ é que se trata de uma estimativa da proporção de variância verdadeira contida na variação total observada na pontuação [25]. Valores médios de 0,71 , sem alteração através da exclusão de cada item do questionário, representam uma pontuação alta, demonstrando que $71 \%$ da variância total observada é verdadeira, indicando alta confiabilidade. Complementando o $\alpha \mathrm{C}$, este estudo avaliou também o CCI, que apresentou valores médios considerados aceitáveis, de 0,705 (IC95\%: 0,634-0,768), demonstrando que o NAKQ possui confiança acima de $70 \%$.

$\mathrm{Na}$ avaliação do teste e reteste essa versão do NAKQ demonstrou possuir reprodutibilidade adequada. $\mathrm{O}$ percentual de concordância atingiu alto nível em 54,84\% das questões e satisfatório em 25,81\% delas, totalizando aproximadamente $75 \%$ de perguntas dentro dos padrões aceitáveis, segundo o índice Kappa. Valores semelhantes foram demonstrados em estudos anteriores [13, 14], possuindo dois itens a mais que o presente estudo, com valores $>0,60$ pontos, não sendo demonstrado ou aplicado pelo estudo original [23].
Quanto à avaliação do nível de conhecimento sobre a asma nos sujeitos estudados, o estudo avaliou se pais de pacientes com asma possuíam maior conhecimento sobre a doença, quando comparados a pais de crianças não asmáticas, analisando se apresentam pontuação mais elevada (validade concorrente). Embora o nível de conhecimento tenha sido abaixo do ponto de corte estipulado pelo NAKQ ( $\geq 21$ pontos) em ambos os grupos, os pais de asmáticos apresentaram escores maiores do que o grupo controle. Em outro estudo [14], demonstrou-se que o grupo de médicos obtiveram a média de escore maior (22,9 pontos) do que o grupo de não médicos $(19,1$ pontos $)(p<0,001)$. Além disso, a pontuação média de profissionais da saúde não médicos, assim como dos pais de asmáticos do presente estudo, foram semelhantes e menores do que 21 pontos, indicando um nível de conhecimento nivelado, diferente dos médicos, que obtiveram pontuação maior do que 21 pontos. Estes dados sugerem que estratégias de educação em asma no Brasil devem ser aprimoradas, particularmente para pais de pacientes com asma e profissionais não médicos da área da saúde.

Como limitação deste estudo encontramos dificuldade na aplicação de outro instrumento com finalidade de análise comparativa dos dados, pois o único instrumento existente e validado no Brasil não é aplicado ao mesmo público.

Concluindo, este estudo oferece à comunidade científica um questionário de avaliação de conhecimento dos pais sobre asma com características psicométricas adequadas e validado para a língua portuguesa no Brasil, podendo ser útil para o planejamento e avaliação de intervenções educativas, melhorando potencialmente estratégias de manejo e controle da asma em crianças.

\section{NOTAS}

\section{Apoio financeiro}

Coordenação de Aperfeiçoamento de Pessoal de Nível Superior (CAPES).

\section{Declaração de conflito de interesses}

Os autores declaram não haver conflitos de interesses relevantes ao conteúdo deste estudo, informam ter tido acesso a todos os dados obtidos e assumem completa responsabilidade pela integridade dos resultados. 


\section{REFERÊNCIAS}

1. Boulet L, FitzGerald J, Reddel H. The revised 2014 GINA strategy report: opportunities for change. Curr Opin Pulm Med. 2015;21(1): 1-7. https://doi.org/10.1097/MCP.0000000000000125

2. Bateman E, Hurd S, Barnes P, Bousquet J, Drazen J, FitzGerald M, Gibson P, Ohta K, O'Byrne P, Pedersen S, Pizzichini E, Sullivan S, Wenzel S, Zar H. Global strategy for asthma management and prevention: GINA executive summary. Eur Respir J. 2008;31(1): 143-78. https://doi.org/10.1183/09031936.00138707

3. Ferkol T, Schraufnagel D. The global burden of respiratory disease. Ann Am Thorac Soc. 2014;11(3):404-6. https://doi.org/10.1513/ AnnalsATS.201311-405PS

4. Zar H, Ferkol T. The global burden of respiratory disease-impact on child health. Pediatr Pulmonol. 2014;49(5):430-4. https://doi. org/10.1002/ppul.23030

5. Castro-Rodriguez J, Custovic A, Ducharme F. Treatment of asthma in young children: evidence-based recommendations. Asthma Res Pract. 2016;2(5):5. https://doi.org/10.1186/s40733-016-0020-z

6. Canonica G, Bagnasco D, Ferrantino G, Ferrando M, Passalacqua G. Update on immunotherapy for the treatment of asthma. Curr Opin Pulm Med. 2016;22(1):18-24. https://doi.org/10.1097/MCP.0000000000000227

7. Volovitz B, Nussinovitch M. Presence and Treatment of Asthma Exacerbation in Infants and Children. Pediat Therapeut. 2016;6(1): 1-6. https://doi.org/10.4172/2161-0665.1000276

8. Riera A, Ocasio A, Tiyyagura G, Thomas A, Goncalves P, Krumeich L, Ragins K, Trevino S, Vaca F. A web-based educational video to improve asthma knowledge for limited English proficiency Latino caregivers. J Asthma. 2016 (just-accepted): 1-8. https://doi.org/ $10.1080 / 02770903.2016 .1251597$

9. Pozo-Beltran C, Navarrete-Rodriguez E, Fernandez-Soto R, Navarro-Munguia J, Hall-Mondragon M, Sienra-Monge J, Del RioNavarro B. Knowledge of asthma: educational intervention with the 2014 GINA guide in primary care physicians. Rev Alerg Mex. 2016;63(4):358-64.

10. Roncada C, Dias C, Sarria E, Pitrez P. Instrumentos específicos para avaliação do conhecimento em asma: revisão sistemática. Medicina (Ribeirao Preto Online). 2015;48(6):619-27. https://doi.org/10.11606/issn.2176-7262.v48i6p619-627

11. Terwee C, Bot S, de Boer M, van der Windt D, Knol D, Dekker J, Bouter L, de Vet H. Quality criteria were proposed for measurement properties of health status questionnaires. J Clin Epidemiol. 2007;60(1):34-42. https://doi.org/10.1016/j.jclinepi.2006.03.012

12. Fitzclarence C, Henry R. Validation of an asthma knowledge questionnaire. J Paediatr Child Health. 1990;26(4):200-4. https://doi. org/10.1111/j.1440-1754.1990.tb02429.x

13. Praena C, Lora E, Aquino L, Sánchez S, Jiménez C. The Spanish version of the Newcastle Asthma Knowledge Questionnaire for parents of children with asthma (NAKQ). Transcultural adaptation and reliability analysis. An Pediatr (Barc). 2009;70(3):209-17. https://doi.org/10.1016/j.anpedi.2008.10.013

14. Cidade S, Roncada C, Costa D, Rafael J, Pitrez P. Validação linguística e psicométrica do questionário sobre conhecimento em asma Newcastle Asthma Knowledge Questionnaire. Rev Ciênc Méd. 2016;24(2):45-54.

15. Globe G, Martin M, Schatz M, Wiklund I, Lin J, von Maltzahn R, Mattera MS. Symptoms and markers of symptom severity in asthma - content validity of the asthma symptom diary. Health Qual Life Outcomes. 2015;13(21):1-9. https://doi.org/10.1186/s12955015-0217-5

16. GINA. Global Strategy for Asthma Management and Prevention [Internet]. Global Initiative for Asthma;2016 [cited 2016 June 07]. Available from: http://ginasthma.org/2016-gina-report-global-strategy-for-asthma-management-and-prevention/

17. Wild D, Grove A, Martin M, Eremenco S, McElroy S, Verjee-Lorenz A, Erikson P, Translation ITFf, Cultural A. Principles of Good Practice for the Translation and Cultural Adaptation Process for Patient-Reported Outcomes (PRO) Measures: report of the ISPOR Task Force for Translation and Cultural Adaptation. Value Health. 2005;8(2):94-104. https://doi.org/10.1111/j.1524-4733.2005.04054.x

18. Cronbach L, Meehl P. Construct validity in psychological tests. Psychol Bull. 1955;52(4):281-302. https://doi.org/10.1037/h0040957

19. Cronbach L. Coefficient alpha and the internal structure of tests. Psychometrika. 1951;16(3):297-334. https://doi.org/10.1007/ BF02310555

20. Maroco J, Garcia-Marques T. Qual a fiabilidade do alfa de Cronbach? Questões antigas e soluções modernas? Laboratório de Psicologia. 2013;4(1):65-90. https://doi.org/10.14417/lp.763

21. Yin H, Gupta R, Mendelsohn A, Dreyer B, van Schaick L, Brown C, Encalada K, Sanchez D, Warren C, Tomopoulos S. Use of a Low Literacy Written Action Plan to Improve Parent Understanding of Pediatric Asthma Management: A Randomized Controlled Study. J Asthma. 2017(Jan 3). https://doi.org/10.1080/02770903.2016.1277542

22. Janevic M, Baptist A, Bryant-Stephens T, Lara M, Persky V, Ramos-Valencia G, Uyeda K, Hazan R, Garrity A, Malveaux F. Effects of pediatric asthma care coordination in underserved communities on parent perceptions of care and asthma-management confidence. J Asthma. 2016(Nov 30):1-6. https://doi.org/10.1080/02770903.2016.1242136

23. Borges M, Ferraz É, Pontes S, Cetlin A, Caldeira R, Silva C, Araújo A, Vianna E. Development and validation of an asthma knowledge questionnaire for use in Brazil. J Bras Pneu. 2010;36(1):8-13. https://doi.org/10.1590/s1806-37132010000100004

24. Varela A, Díaz S, Esteban S, Murúa J, López B, Martínez-Gimeno A. Validación de un cuestionario en castellano sobre conocimientos de asma en profesores. Arch Bronconeumol. 2015;51(3):115-20. https://doi.org/10.1016/j.arbres.2014.01.015

25. Adánez G, González A. Fiabilidad y validez. Papeles del psicólogo. 2010;31(1):67-74. 